\title{
ACADEMIC POSITION IN MEDICAL EDUCATION RESEARCH
}

The Office of Post Graduate Medical Education (PGME), together with the Medical Education and Research Unit (MERU), invites applications for a full-time academic position in medical education research at the Assistant or Associate Professor level.

Responsibilities include the promotion and development of collaborative educational and assessment tools and innovation in residency programs, educational research within the postgraduate medical education residency programs as well as the facilitation of resident and faculty participation in postgraduate medical education and the development of academic careers in medical education. Responsibilities also include the teaching of graduate courses as well as the supervision of MSc and PhD students in medical education.

The successful candidate will have responsibilities for educational evaluation and research in PGME and will be available to clinician educators who need consultation and support in initiating research ideas. Research may be stimulated by a number of mechanisms such as workshops on research methods, grant writing, poster days, consultation, and active participation as co-investigator with input to grant writing, data gathering, analysis and publication. As a faculty member, the candidate will maintain an active research program including research grants, publications, etc. and will have the usual responsibility for teaching and supervision of clinical fellows and graduate students.

Qualifications include a PhD in medical education or the social and behavioural sciences and a strong background in quantitative methods, psychometrics, and statistics (or MD with expertise in medical education in these areas). Demonstrated competence in conducting research and publication will be an asset.

Please forward your curriculum vitae, statement of research interests, and names of at least three referees, by August 31, 2010, to:

Dr. Claudio Violato,

Professor and Director, Medical Education and Research Unit

Department of Community Health Sciences

Faculty of Medicine, University of Calgary

3330 Hospital Drive N.W

Calgary, AB, Canada T2N 4NI
All qualified candidates are encouraged to apply; however, Canadian citizens and permanent residents will be given priority. The University of Calgary respects, appreciates, and encourages diversity. 